\title{
Sequential adjuvant chemotherapy and radiotherapy in endometrial cancer - Results from two randomised studies
}

\author{
Thomas Hogberg ${ }^{a, *}$, Mauro Signorelli ${ }^{b}$, Carlos Freire de Oliveira ${ }^{c}$, Roldano Fossati ${ }^{d}$, \\ Andrea Alberto Lissoni ${ }^{e}$, Bengt Sorbe ${ }^{f}$, Håkan Andersson ${ }^{g}$, Seija Grenman ${ }^{h}$, \\ Caroline Lundgren ${ }^{i}$, Per Rosenberg ${ }^{j}$, Karin Boman ${ }^{k}$, Bengt Tholander ${ }^{l}$, \\ Giovanni Scambia ${ }^{m}$, Nicholas Reed ${ }^{n}$, Gennaro Cormio ${ }^{\circ}$, Germana Tognon ${ }^{p}$, \\ Jackie Clarke ${ }^{q}$, Tomasz Sawicki ${ }^{r}$, Paolo Zola ${ }^{s}$, Gunnar Kristensen ${ }^{t}$
}

a Department of Cancer Epidemiology, University of Lund, University Hospital, SE-221 85 Lund, Sweden

${ }^{\mathrm{b}}$ Department of Obstetrics and Gynaecology, A. Manzoni Hospital, Lecco, Italy

${ }^{\mathrm{c}}$ Department of Gynaecology, University Hospital, Coimbra, Portugal

d Department of Oncology, Mario Negri Institute, Milan, Italy

e Department of Gynaecology and Obstetrics, S. Gerardo Hospital, Monza, Italy

${ }^{\mathrm{f}}$ Department of Gynaecological Oncology, University Hospital, Örebro, Sweden

g Department of Gynaecological Oncology, University Hospital, Gothenburg, Sweden

${ }^{\mathrm{h}}$ Department of Obstetrics and Gynaecology, University Hospital, Turku, Finland

${ }^{i}$ Department of Gynaecological Oncology, Radiumhemmet, Karolinska Hospital Solna, Stockholm, Sweden

${ }^{j}$ Department of Gynaecological Oncology, University Hospital, Linköping, Sweden

${ }^{\mathrm{k}}$ Department of Gynaecological Oncology, University Hospital, Umeå, Sweden

${ }^{1}$ Department of Oncology, University Hospital, Uppsala, Sweden

$\mathrm{m}$ Department of Obstetrics and Gynaecology, Gemelli Hospital, Rome, Italy

${ }^{\mathrm{n}}$ Beatson Oncology Centre, Western Infirmary, Glasgow, UK

- Department of Obstetrics and Gynaecology, University of Bari, Bari, Italy

$\mathrm{p}$ Department of Obstetrics and Gynaecology, Spedali Civili, Brescia, Italy

${ }^{a}$ Belfast City Hospital, Belfast, UK

${ }^{\mathrm{r}}$ Department of Oncology and Radiotherapy, Medical University of Gdansk, Gdansk, Poland

s Department of Obstetrics and Gynaecology, Mauriziano Hospital, Turin, Italy

${ }^{\mathrm{t}}$ Department of Gynaecological Oncology and Institute of Medical Informatics, The Norwegian Radium Hospital, Oslo, Norway

\section{A R T I C L E I N F O}

Article history:

Received 21 April 2010

Received in revised form 26 May

2010

Accepted 2 June 2010

Available online 7 July 2010

Keywords:

Adjuvant therapy

Chemotherapy

\section{A B S T R A C T}

Introduction: Endometrial cancer patients with high grade tumours, deep myometrial invasion or advanced stage disease have a poor prognosis. Randomised studies have demonstrated the prevention of loco-regional relapses with radiotherapy (RT) with no effect on overall survival (OS). The possible additive effect of chemotherapy (CT) remains unclear. Two randomised clinical trials (NSGO-EC-9501/EORTC-55991 and MaNGO ILIADE-III) were undertaken to clarify if sequential combination of chemotherapy and radiotherapy improves progression-free survival (PFS) in high-risk endometrial cancer. The two studies were pooled.

Methods: Patients ( $n=540 ; 534$ evaluable) with operated endometrial cancer International Federation of Obstetrics and Gynaecology (FIGO) stage I-III with no residual tumour and

\footnotetext{
* Corresponding author: Tel.: +46 46 177550; fax: +46 46188143.

E-mail address: thomas.hogberg@med.lu.se (T. Hogberg).

0959-8049/\$ - see front matter (c) 2010 Elsevier Ltd. All rights reserved.

doi:10.1016/j.ejca.2010.06.002
} 
Radiotherapy

Chemoradiotherapy

Endometrial cancer

Randomised clinical trial prognostic factors implying high-risk were randomly allocated to adjuvant radiotherapy with or without sequential chemotherapy.

Results: In the NSGO/EORTC study, the combined modality treatment was associated with $36 \%$ reduction in the risk for relapse or death (hazard ratio (HR) $0.64,95 \%$ confidence interval (CI) 0.41-0.99; $P=0.04$ ); two-sided tests were used. The result from the Gynaecologic Oncology group at the Mario Negri Institute (MaNGO)-study pointed in the same direction (HR 0.61), but was not significant. In the combined analysis, the estimate of risk for relapse or death was similar but with narrower confidence limits (HR 0.63, CI 0.44-0.89; $P=0.009$ ). Neither study showed significant differences in the overall survival. In the combined analysis, overall survival approached statistical significance (HR 0.69, CI 0.46-1.03; $P=0.07$ ) and cancer-specific survival (CSS) was significant (HR 0.55, CI 0.35-0.88; $P=0.01$ ).

Conclusion: Addition of adjuvant chemotherapy to radiation improves progression-free survival in operated endometrial cancer patients with no residual tumour and a high-risk profile. A remaining question for future studies is if addition of radiotherapy to chemotherapy improves the results.

(C) 2010 Elsevier Ltd. All rights reserved.

\section{Introduction}

Endometrial cancer is the most common gynaecologic cancer in the Western world. It was estimated that worldwide around 200,000 women acquired and 50,000 died of endometrial cancer in 2002. ${ }^{1}$ The prognosis for early-stage endometrial cancer is excellent, but subgroups with a high risk for micrometastatic disease have been identified. ${ }^{2}$ Randomised studies demonstrate high loco-regional control in early-stage endometrial cancer with adjuvant pelvic external radiotherapy (RT).$^{3-6}$ However, overall survival (OS) remains largely unaffected. It is therefore likely that patients at risk for micrometastatic disease will benefit from systemic adjuvant therapy.

The Nordic Society of Gynaecological Oncology/European Organisation for the Research and Treatment of Cancer (NSOG/EORTC) trial was designed to investigate if the addition of systemic chemotherapy (CT) to pelvic RT would improve progression-free survival (PFS) and OS for patients with endometrial cancer at high risk for micrometastatic disease. After presentation of the preliminary results at the American Society of Clinical Oncology (ASCO) $2007^{7}$ it was decided to publish the study together with the results from a similar trial (ILIADE-III) performed by the Gynaecological Oncology group at the Mario Negri Institute (MaNGO). The results of the ILIADE-III were not known.

When these studies were planned Thigpen and colleagues had presented their randomised trial of doxorubicin + cisplatin versus doxorubicin at ASCO 1993. ${ }^{8}$ This regimen was chosen in both studies.

We report the results of the NSGO/EORTC and the MaNGOtrials and an analysis of the pooled data.

\section{Materials and methods}

\subsection{The NSGO-9501/EORTC-55991 trial}

The inclusion criteria were histologically verified endometrial cancer, surgery with total abdominal hysterectomy and bilateral salpingo-oophorectomy (lymphadenectomy (LA) was optional), no residual postoperative macroscopic tumour,
International Federation of Obstetrics and Gynaecology (FIGO) 1988 surgical stage I, age $\leqslant 80$ years, World Health Organisation performance status $<3$ and adequate bone marrow, liver and kidney function. The risk assessment was based on FIGO stage, grade and myometrial invasion. Most Swedish departments also used DNA ploidy. Patients were eligible if they had a risk profile that qualified for adjuvant treatment. Patients with serous, clear cell or anaplastic carcinomas were eligible regardless of other risk factors. Exclusion criteria were: para-aortic lymph node involvement, squamous carcinoma or small cell carcinoma with neuroendocrine differentiation, pre-operative irradiation and previous or concurrent malignant disease except for curatively treated carcinoma in situ of the cervix or basal cell carcinoma of the skin.

Amendment 1 August 2002 (237 patients included) allowed inclusion of patients with FIGO 1988 occult stage II, stage IIIA (only positive peritoneal fluid cytology) and stage IIIC (only positive pelvic lymph nodes without postoperative macroscopic residual tumour).

Randomisation was performed centrally by the study office at Linkoping University Hospital for NSGO patients and at the EORTC Headquarters for EORTC patients. NSGO patients were randomised in blocks with stratification for centre and histology. The EORTC used a minimisation procedure with the same stratification factors. ${ }^{9}$

Pelvic RT was given according to departmental guidelines ( $\geqslant 44 \mathrm{~Gy}$ ). RTwas given before CT in the sequential radiotherapy and chemotherapy (RT-CT)-arm. Optional vaginal brachytherapy had to be decided before randomisation. Amendment 1 allowed the choice of sequence of RT and CT before randomisation. CT consisted of four courses of doxorubicin/epirubicin $50 \mathrm{mg} / \mathrm{m}^{2}+$ cisplatin $50 \mathrm{mg} / \mathrm{m}^{2}$ every 4 weeks. Amendment 2 on August 2004 (291 patients included) allowed alternative CT regimens, including: paclitaxel $175 \mathrm{mg} / \mathrm{m}^{2}+$ epirubicin $60 \mathrm{mg} / \mathrm{m}^{2}$ or doxorubicin $40 \mathrm{mg} / \mathrm{m}^{2}+$ carboplatin AUC 5 or paclitaxel $175 \mathrm{mg} / \mathrm{m}^{2}$ + carboplatin AUC 5-6 every 3 weeks.

Patients were followed at 3 and 6 months after treatment and thereafter every 6 months for 5 years. A gynaecological examination was performed at each visit. A chest X-ray was to be taken annually. 


\subsection{The MaNGO ILIADE-III study}

In 1996, the MaNGO group started the multicentre ILIADEstudy in endometrial cancer, which consisted of three protocols. ILIADE-I investigated different techniques for hysterectomy, ${ }^{10}$ ILIADE-II the question of $\mathrm{LA}^{11}$ and ILIADE-III adjuvant therapy.

The inclusion criteria for ILIADE-III were histologically confirmed endometrioid carcinoma, FIGO 1988 stage IIB, IIIA-C disease (stage IIIA with positive cytology alone without other risk factors was not included). Exclusion criteria: serous/clear cell carcinomas, performance status $>2$, previous malignancy except for basal cell carcinoma of the skin, surgical procedures less than total abdominal hysterectomy and bilateral salpingo-oophorectomy (LA was optional), previous hormonal/chemo/radiotherapy for the present tumour, impaired cardiac function, evidence of any other serious disease and inadequate bone marrow, liver or kidney function.

Patients were randomised in blocks that balanced the treatment assignment within each site. Randomisation was performed centrally by telephone at the Mario Negri Institute, Milan.

CT had to start within 30 days after surgery and consisted of doxorubicin $60 \mathrm{mg} / \mathrm{m}^{2}$ + cisplatin $50 \mathrm{mg} / \mathrm{m}^{2}$ every 3 weeks for three cycles. The interval between CT and RT had to be less than 4 weeks, while patients allocated to RT alone had to start within 40 days after surgery. Pelvic RT was given with $1.8 \mathrm{~Gy}$ fractions; total dose $45 \mathrm{~Gy}$. For patients with para-aortal metastases, a para-aortal field was added up to L1/L2. Vaginal brachytherapy was added for women with cervical stromal involvement.

Patients were monitored every 3-4 months during the first 2 years, every 6 months for the next 3 years and then annually. The protocol recommended yearly computer tomography or ultrasound of the pelvis and abdomen for the first 3 years.

The study protocols were reviewed and approved by the local ethics committees. Informed consent was obtained from all patients. Patients and staff were not blinded to the treatment assignment.

\subsection{Statistics}

The primary end-point was PFS. All times were counted from the time of randomisation. PFS was defined as the time to progression of endometrial cancer or death from all causes. Secondary end-points were OS; the time to death of all causes and cancer-specific survival (CSS); the time to death related to endometrial cancer.

Both studies aimed at detecting a $15 \%$ absolute improvement in 5 year PFS from $60 \%$ to $75 \%$. Assuming exponential survival distributions this corresponds to a hazard ratio (HR) of 0.56 . Because of different assumptions about inclusion and follow-up the number of patients in the NSGO/EORTC and the MaNGO-trials was predetermined to 400 and 300, respectively. The power calculation in the NSGO/EORTC-study was based on OS.

The NSGO/EORTC and MaNGO databases were locked 19th August 2009 and 6th March 2008, respectively. The intentionto-treat principle was used in the analyses after exclusion of five patients in the NSGO/EORTC-study (Fig. 1a) and one pa- tient with stage IV disease in the MaNGO-trial (Fig. 1b). Survival curves were constructed by the Kaplan-Meier technique. Survival differences between groups were expressed as hazard ratios and were analysed with univariate Cox proportional hazard models ${ }^{12}$ with stratification for department. Departments which included less than four patients were aggregated within EORTC $(n=6)$ and MaNGO $(n=9)$, respectively; all sites in the NSGO randomised four or more patients. We also made a supportive Cox proportional hazard model with age, stage, grade and cell type as covariates to check if the treatment effect was affected. To analyse potential heterogeneity of the treatment effect over subgroups, the interaction between treatment effect and group variable was evaluated and illustrated with forest plots. ${ }^{13}$ Potential heterogeneity among study groups and after amendment 1 and 2 in the NSGO/EORTC-trial was analysed with Cox-models and illustrated in a forest plot. Two-sided tests were used for significance testing. We used Stata Version 10 (StataCorp, TX, USA).

\section{Results}

Between May 1996 and January 2007, 383 patients were randomised in the NSGO/EORTC study, 320 from 13 NSGO departments and 63 from 12 EORTC departments (Fig. 1a). In the MaNGO-study, 157 patients from 20 departments were randomised between October 1998 and July 2007 (Fig. 1b). The treatment arms were well balanced regarding prognostic factors (Table 1).

Whether LA was performed was registered in EORTC patients and after amendment 2 in the NSGO. Twenty-eight out of 61 patients in the RT-arm (46\%) had a pelvic LA; eight patients also underwent para-aortic LA. In the RT-CT-arm 35/72 (49\%) underwent pelvic LA; six also underwent paraaortic LA. In the MaNGO-trial 41/76 (54\%) underwent systematic pelvic LA in the RT-arm; seven (9.2\%) also had low paraaortal and six (7.9\%) high para-aortal LA. While 36/80 (45\%) in the RT-CT-arm underwent systematic pelvic LA; seven (8.8\%) and three (3.8\%) had additional low or high para-aortic LA (Table 1).

The compliance to RT was high in the NSGO/EORTC-study, $182 / 191$ (95\%) and 178/187 (95\%) received $\geqslant 44$ Gy in the RTarm and RT-CT-arm, respectively. Of the 187 patients assigned to CT, 136 (73\%) received four treatment cycles as planned. Eighteen (9.6\%) received no CT and the CT data were not available for three patients (1.6\%) (Fig. 1a). Vaginal brachytherapy was used in 75/191 (39\%) of the cases in the RT-arm and $82 / 187$ (44\%) in the RT-CT-arm (Table 1).

Most patients $(138 / 166,83 \%)$ received doxorubicin/epirubicin + cisplatin, six patients $(3.6 \%)$ epirubicin + carboplatin, five (3\%) paclitaxel + epirubicin + carboplatin and $17(10 \%)$ paclitaxel + carboplatin. Only $28(17 \%)$ had CT before RT and the sequence is unknown for seven (4\%).

Eight patients (5.1\%) in the MaNGO-trial did not undergo RT. Patients assigned to RT or RT-CT received the same median pelvic RT dose (50 Gy). Seventy-one out of 80 patients (89\%) completed three courses of CT, three (3.8\%) received less than three courses, two (2.5\%) did not start CT because of patients' refusals and CT data were missing for four patients 


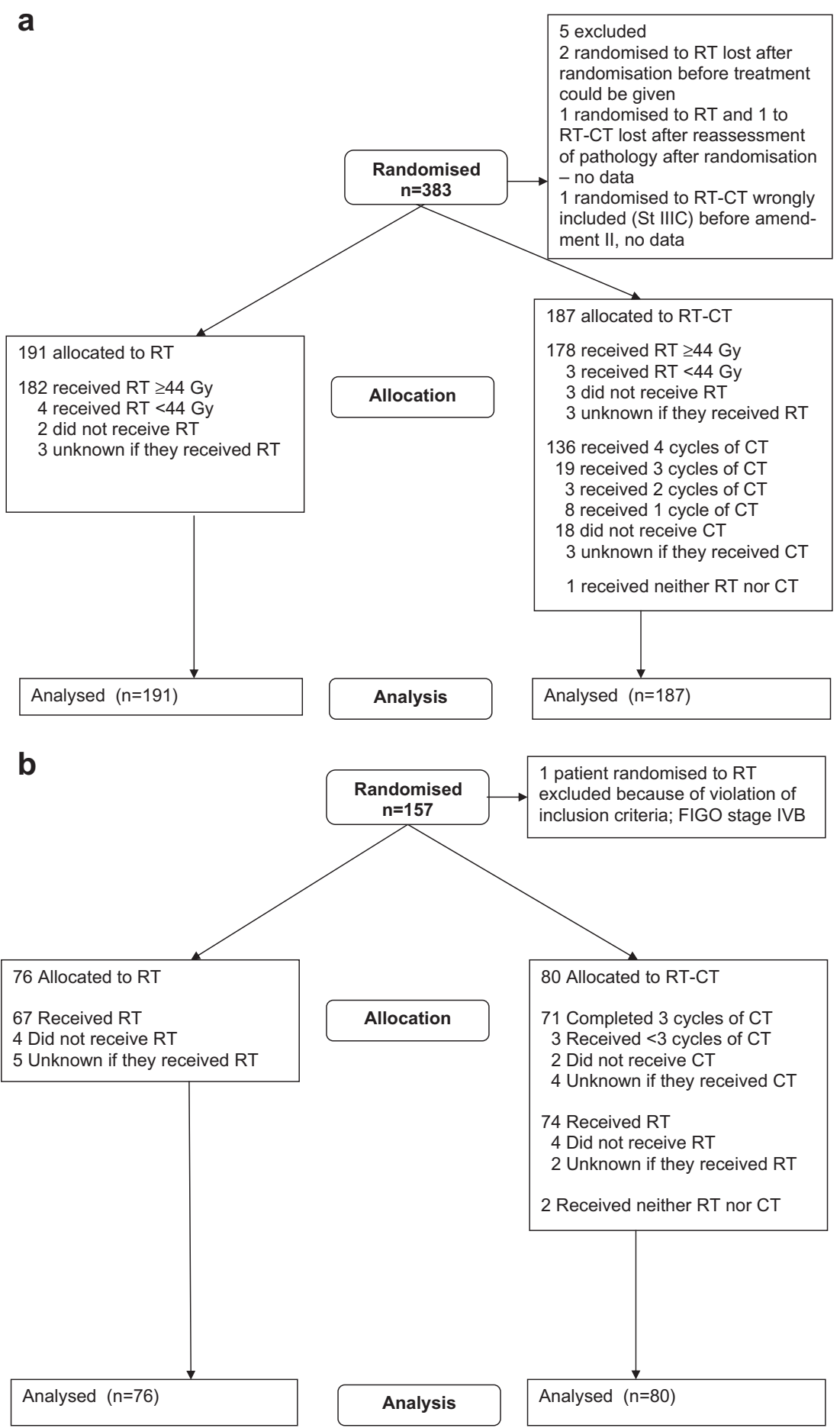

Fig. 1 - (a) Consort flowchart NSGO/EORTC-study. (b) Consort flowchart ILIADE-study.

(5.0\%) (Fig. 1b). In the RT-arm 21/76 (28\%) received vaginal brachytherapy. The corresponding figure in the RT-CT-arm was $25 / 80(31 \%)$ (Table 1$)$.

In the NSGO/EORTC-trial, there was one treatment-related death 3 months after randomisation in the RT-arm. No further details were available. There were eight serious adverse events (SAE) in the RT-CT-arm: two cases with diarrhoea, one combined with neutropenia; three events with neutropenia one with pneumonia requiring respirator treatment, and another with associated nausea and vomiting; one patient with allergic reaction to paclitaxel; one case with an episode of atrial fibrillation; and one patient with bilateral pulmonary emboli 24 days after cycle one. There was one SAE in the RTarm; an intestinal reaction with diarrhoea which led to cessation of RT after $36 \mathrm{~Gy}$. All SAE's resolved after appropriate treatment.

In the MaNGO-trial no treatment-related death was registered. Analysis of toxicity was performed in 74 patients 


\section{Table 1 - Patient characteristics.}

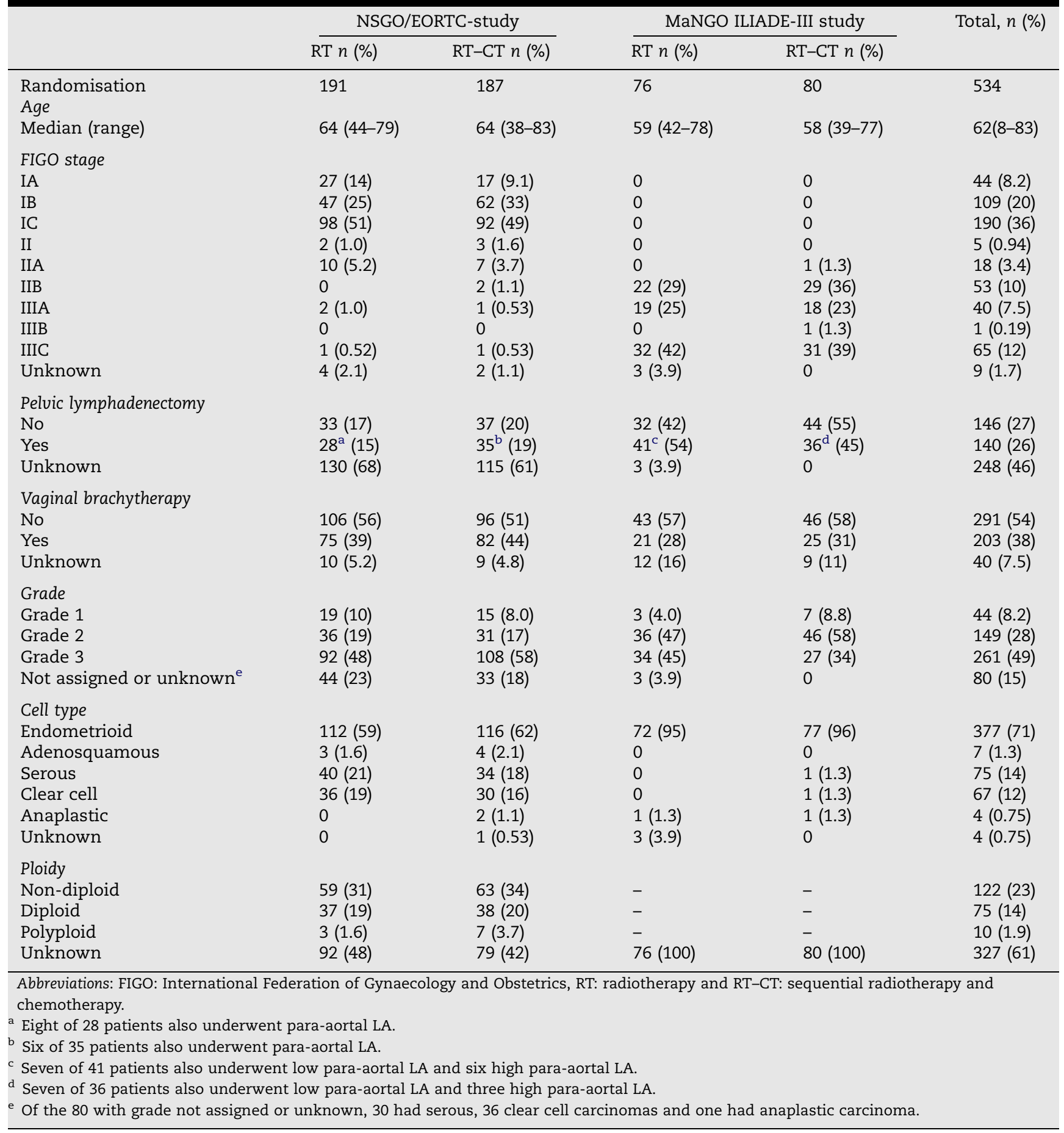

receiving at least one course of $\mathrm{CT}$. The median cisplatin and doxorubicin doses per cycle were 50 (25th-75th percentiles $=49-50)$ and $60(25 \mathrm{th}-75 \mathrm{th}$ percentiles $=56-60) \mathrm{mg} / \mathrm{m}^{2}$, respectively. The maximum grades of toxicities observed during treatment were: grade 3/4 leucopenia in 12 patients $(16 \%)$; grade $3 / 4$ neutropenia in $22(30 \%)$; grade 2 thrombocytopenia in seven (9\%); grade 2 anaemia in seven (9\%); grade $3 / 4$ nausea and vomiting in four (5\%); and grade $2 / 3$ alopecia in $37(50 \%)$.

Disease progression was registered in 46/191 (24\%) and 28/ 187 (15\%) patients in the RT- and RT-CT-arm, respectively in the NSGO/EORTC-study. The corresponding figures for the MaNGO-trial were 24/76 (32\%) and 15/80 (19\%). Table 2 shows the progression sites.

The difference in PFS between the treatment groups in the NSGO/EORTC-trial was significant, favouring RT-CT with, HR 0.64 (95\% CI 0.41-0.99) $P=0.04$ (Table 3). In the MaNGO-trial we found a non-significant difference of about the same magnitude (HR 0.61) (Table 3). When pooling the data from both studies there was a highly significant difference favouring RT-CTwith HR 0.63 (95\% CI 0.41-0.99) P = 0.009 (Fig. 2, Table 3). 


\section{Table 2 - Sites of progression.}

\begin{tabular}{llc} 
& RTn (\%) & RT-CTn (\%) \\
\hline Loco-regional & $11(16)$ & $5(12)$ \\
Distant & $52(74)$ & $35(81)$ \\
Unknown/multiple sites & $7(10)$ & $3(7.0)$ \\
Total & $70(100)$ & $43(100)$ \\
\hline $\begin{array}{l}\text { Abbreviations: RT: radiotherapy and RT-CT: sequential radiotherapy } \\
\text { and chemotherapy. }\end{array}$ \\
\hline
\end{tabular}

Neither the NSGO/EORTC nor the MaNGO-trial (Table 3) showed significant differences in OS. The analysis of the pooled data approached statistical significance with HR 0.69 (95\% CI 0.46-1.03) $P=0.07$ (Fig. 3, Table 3). The OS curves are almost equal up to about 2 years and then they tend to split up in favour of RT-CT.

The difference favouring RT-CT was significant for CSS in the NSGO/EORTC-trial with HR 0.51 (95\% CI 0.28-0.90) $P=0.02$, but not in the MaNGO-trial (HR 0.65). There was a significant difference in the pooled data favouring RT-CT with HR 0.55 (95\% CI 0.35-0.88) P=0.01 (Table 3).

A Cox proportional hazard model on 447 patients with no missing values in any of the covariates (214 randomised to RT and 233 to RT-CT) with age, stage, grade and cell type as covariates, stratification for department and PFS as the endpoint demonstrated that the treatment effect was stable after adjustment for prognostic factors. The HR was 0.65 (95\% CI
0.43-0.99) compared to 0.63 (95\% CI 0.42-0.93) without adjustment for covariates. The analysis of heterogeneity of treatment effect on PFS in patient subgroups was performed on the same patients as the Cox model above, except that three further patients with anaplastic/undifferentiated tumours were excluded. LA could only be analysed in the subset where this was registered $(n=286)$. There was no evidence of heterogeneity of treatment effect with regard to age, grade, stage, cell type or LA (Fig. 4). Fig. 5 shows another forest diagram exploring if there was heterogeneity between study groups and amendments in the NSGO/EORTC trial. As can be seen the treatment effect is similar.

The apparent lack of effect in serous and clear cell carcinomas led to an unplanned data-driven subgroup analysis of endometrioid carcinomas in the pooled population $(n=384)$. For PFS, the HR was 0.53 (95\% CI 0.34-0.83) $P=0.005$ which translates to $11 \%$ absolute difference in 5-year survival from $69 \%$ to $80 \%$ favouring RT-CT. Even OS was of borderline significance, HR 0.60; $P=0.05$ (Table 3). For the 140 patients with serous or clear cell carcinoma in the NSGO/EORTC-study, the HR for PFS was 0.83 (95\% CI 0.42-1.64) $P=0.59$ (Table 3).

\section{Discussion}

The NSGO/EORTC-trial showed that the sequential addition of CT to RT was associated with a significant $36 \%$ reduction in the risk of relapse or death and a significant $49 \%$ reduction

\section{Table 3 - Results of survival analyses in different groups.}

\begin{tabular}{|c|c|c|c|c|c|c|c|c|c|c|}
\hline \multirow[t]{2}{*}{ End-point } & \multicolumn{5}{|c|}{ Events } & \multirow[t]{2}{*}{ HR } & \multirow[t]{2}{*}{$95 \%$ CI } & \multirow[t]{2}{*}{$P$} & \multicolumn{2}{|c|}{ 5-Year probability of surviva } \\
\hline & RT & $\%$ & RT-CT & $\%$ & Total & & & & RT & RT-CT \\
\hline \multicolumn{11}{|c|}{ NSGO-EC-9501/EORTC-55991 (RT $n=191$, RT-CT $n=187)$} \\
\hline PFS & 50 & 26 & 35 & 19 & 85 & 0.64 & $0.41-0.99$ & 0.04 & 0.72 & 0.79 \\
\hline OS & 40 & 21 & 28 & 15 & 68 & 0.66 & $0.40-1.08$ & 0.10 & 0.76 & 0.83 \\
\hline CSS & 34 & 18 & 19 & 10 & 53 & 0.51 & $0.28-0.90$ & 0.02 & 0.79 & 0.88 \\
\hline \multicolumn{11}{|c|}{ MaNGO ILIADE-III (RT $n=76$, RT-CT $n=80)$} \\
\hline PFS & 26 & 34 & 18 & 23 & 44 & 0.61 & $0.33-1.12$ & 0.10 & 0.61 & 0.74 \\
\hline Os & 17 & 22 & 14 & 18 & 31 & 0.74 & $0.36-1.52$ & 0.41 & 0.73 & 0.78 \\
\hline CSS & 15 & 20 & 11 & 14 & 26 & 0.65 & $0.30-1.44$ & 0.29 & 0.76 & 0.82 \\
\hline \multicolumn{11}{|c|}{ POOLED NSGO-EC-9501/EORTC-55991 + MaNGO ILIADE-III (RT $n=267$, RT-CT $n=267)$} \\
\hline PFS & 76 & 28 & 53 & 20 & 129 & 0.63 & $0.44-0.89$ & 0.009 & 0.69 & 0.78 \\
\hline OS & 57 & 21 & 42 & 16 & 99 & 0.69 & $0.46-1.03$ & 0.07 & 0.75 & 0.82 \\
\hline CSS & 49 & 18 & 30 & 11 & 79 & 0.55 & $0.35-0.88$ & 0.01 & 0.78 & 0.87 \\
\hline \multicolumn{11}{|c|}{ NSGO-EC-9501/EORTC-55991 endometrioid carcinoma (RT $n=115$, RT-CT $n=120)$} \\
\hline PFS & 29 & 25 & 19 & 16 & 48 & 0.50 & $0.27-0.95$ & 0.03 & 0.73 & 0.83 \\
\hline OS & 25 & 22 & 15 & 13 & 40 & 0.55 & $0.28-1.09$ & 0.08 & 0.75 & 0.86 \\
\hline CSS & 22 & 19 & 11 & 9 & 33 & 0.42 & $0.19-0.93$ & 0.03 & 0.76 & 0.92 \\
\hline \multicolumn{11}{|c|}{ NSGO-EC-9501/EORTC-55991 serous and clear cell carcinoma (RT $n=76$, RT-CT $n=64)$} \\
\hline PFS & 21 & 28 & 16 & 25 & 37 & 0.83 & $0.42-1.64$ & 0.59 & 0.71 & 0.72 \\
\hline OS & 15 & 20 & 13 & 20 & 28 & 0.94 & $0.42-2.08$ & 0.88 & 0.78 & 0.77 \\
\hline CSS & 12 & 16 & 8 & 13 & 20 & 0.71 & $0.26-1.90$ & 0.49 & 0.82 & 0.85 \\
\hline \multicolumn{11}{|c|}{ POOLED NSGO-EC-9501/EORTC-55991 + MaNGO ILIADE-III endometrioid carcinoma $($ RT $n=187$, RT-CT $n=197)$} \\
\hline PFS & 54 & 29 & 35 & 18 & 89 & 0.53 & $0.34-0.83$ & 0.005 & 0.69 & 0.80 \\
\hline OS & 41 & 22 & 27 & 14 & 68 & 0.60 & $0.36-1.00$ & 0.05 & 0.74 & 0.84 \\
\hline CSS & 36 & 19 & 21 & 11 & 57 & 0.51 & $0.29-0.91$ & 0.02 & 0.77 & 0.87 \\
\hline
\end{tabular}

Abbreviations: CI: confidence interval, CSS: cancer-specific survival, HR: hazard ratio, OS: overall survival, PFS: progression-free survival, RT: radiotherapy and $\mathrm{RT}-\mathrm{CT}$ : sequential radiotherapy and chemotherapy. 


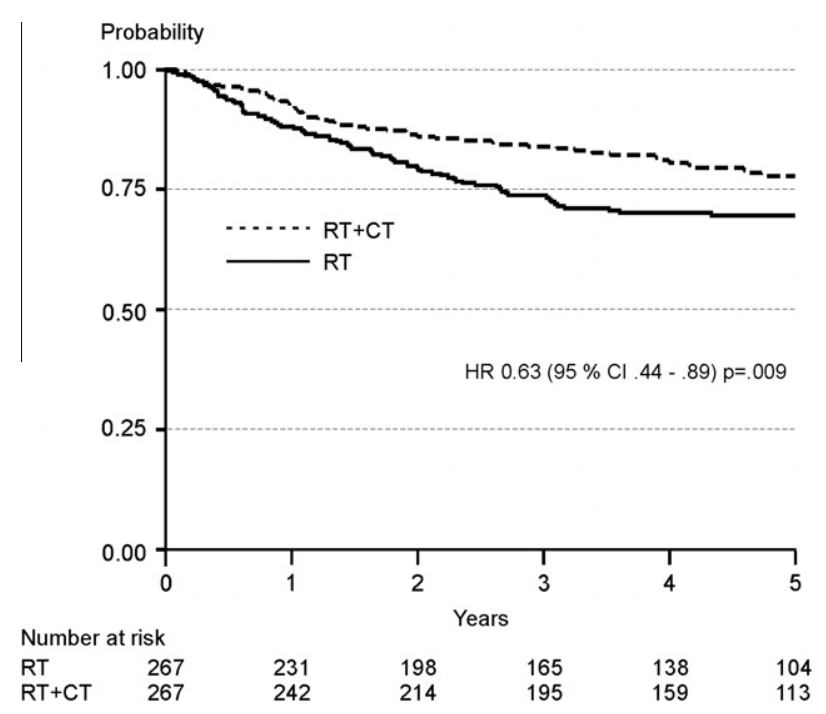

Fig. 2 - Progression-free survival in the pooled NSGO-EC9501/EORTC-5591 and MaNGO studies (CI: confidence interval, HR: hazard ratio, RT: radiotherapy and $\mathrm{RT}-\mathrm{CT}$ : sequential radiotherapy and chemotherapy).

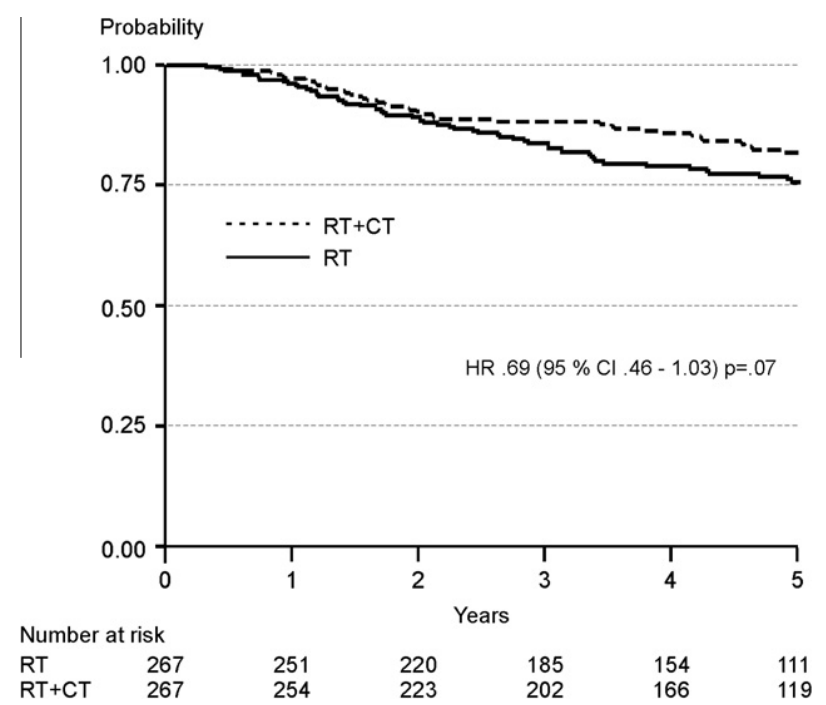

Fig. 3 - Overall survival in the pooled NSGO-EC-9501/EORTC5591 and MaNGO studies (CI: confidence interval, HR: hazard ratio, $\mathrm{RT}$ : radiotherapy and $\mathrm{RT}-\mathrm{CT}$ : sequential radiotherapy and chemotherapy).

in the risk of death from endometrial cancer. The results in the MaNGO-trial point in the same direction but are not significant, likely because of the small study population. The NSGO/EORTC- and MaNGO-trials addressed the same question but in slightly different patient groups. The NSGO/ EORTC-trial initially included only patients with FIGO stage I disease, but later also allowed inclusion of stage II and III. However, relatively few patients with higher stages were included. The MaNGO-trial included patients with more advanced stage disease (FIGO stage II-III). Serous/clear cell carcinomas were included in the NSGO/EORTC-trial while they were excluded in ILIADE. Otherwise, these two randomised studies were fairly similar and it seemed reasonable to pool the data to increase the statistical power and get a more representative stage distribution. With pooled data the estimates were similar but with narrower confidence limits. The 31\% risk reduction of death from any cause in the pooled data still only approached statistical significance. Endometrial cancer mainly affects elderly women and the risk of death due to intercurrent disease is fairly high. There was a significant $45 \%$ risk reduction when looking at cancer-specific survival (CSS).

Endometrial cancer is a radiosensitive tumour. Adjuvant external RT prevents the majority of pelvic disease progressions, but many patients still die of distant metastatic disease. ${ }^{3-6,14}$ It has long been obvious that an effective systemic adjuvant therapy should be added to or replace adjuvant RT. The first randomised study (GOG-34) on adjuvant CT in endometrial cancer was initiated by the US Gynecologic Oncology Group (GOG) already in $1977 .{ }^{15}$ After adjuvant pelvic external RT, patients were randomised to observation or to receive doxorubicin. The study was terminated prematurely because of slow recruitment and no significant difference in OS or PFS could be found between the treatment arms.

GOG-122 included 396 evaluable patients with FIGO stage III or IV endometrial carcinoma of any histology who after surgery were randomised to CT (eight cycles of a doublet regimen containing doxorubicin + cisplatin) or whole abdominal RT. ${ }^{16}$ Both OS and PFS were significantly better for patients in the CT arm. However, this was not a pure study of adjuvant therapy since $16 \%$ of the patients had residual postoperative tumours $<2 \mathrm{~cm}$.

In contrast, two other randomised studies, comparing RT against CT, failed to show superiority of adjuvant CT versus $\mathrm{RT}$ in terms of both disease-free survival and OS. ${ }^{17,18}$ RT was compared with a three-drug regimen (cyclophosphamide + doxorubicin + cisplatin). Maggi and collegues ${ }^{17}$ suggested that the two modalities may be complementary as RT seemed to achieve better loco-regional control of the disease, while CT seemed to better control the distant spread. The main difference between these trials and our study is that we combined RT and CT in the experimental arm.

Limitations of our trials must be acknowledged. (1) The eligibility criteria allowed inclusion of patients with several risk levels of the disease. Although the 5-year survival rates in the control arm were consistent with other similar trials ${ }^{4-6,17,18}$ the prognostic profile of many patients was rather favourable and this might have reduced the statistical power. (2) We used different CT regimens. However, all were well validated for the salvage treatment of endometrial cancer. The majority (90\%) received a combination of anthracycline and platinum. The aim of the studies was to find out if systemic therapy added to RT could improve survival and not to evaluate the efficacy of any specific regimen. We thought it appropriate to allow different regimens to increase inclusion rate. (3) Quality of life data were only registered in some of the patients and have not been analysed. (4) Lympadenectomy was optional and was only registered and performed in a fraction of the patients.

A supportive Cox analysis of the pooled material from the NSGO/EORTC and MaNGO showed that the treatment effect on PFS remained unaffected with age, stage, grade and cell type as covariates and we found no evidence that the PFS benefit for RT-CT compared with RT differed within subgroups (stage 
Age

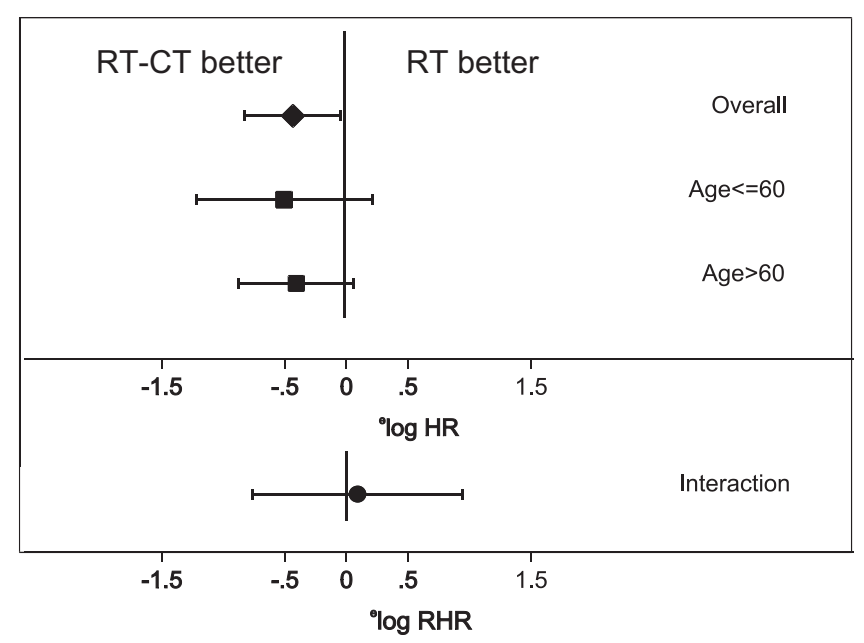

Grade

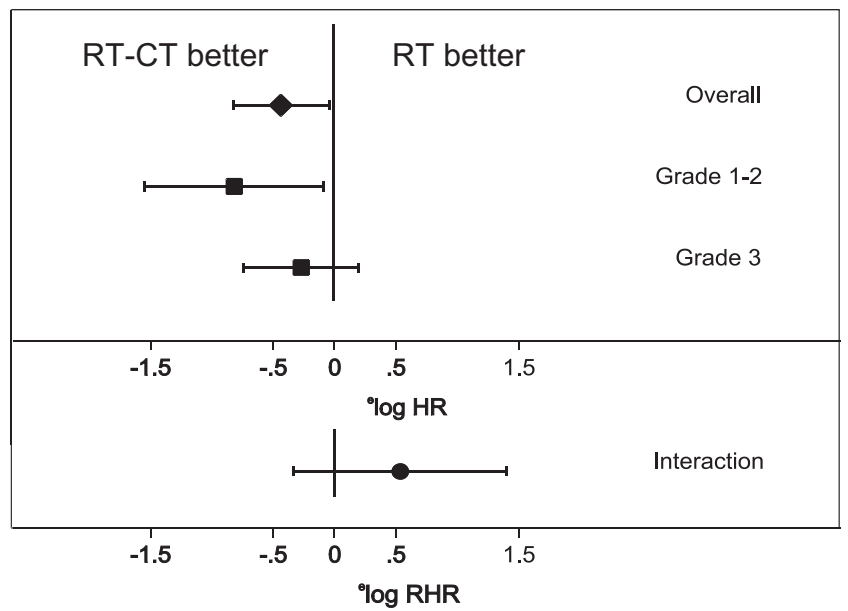

Stage

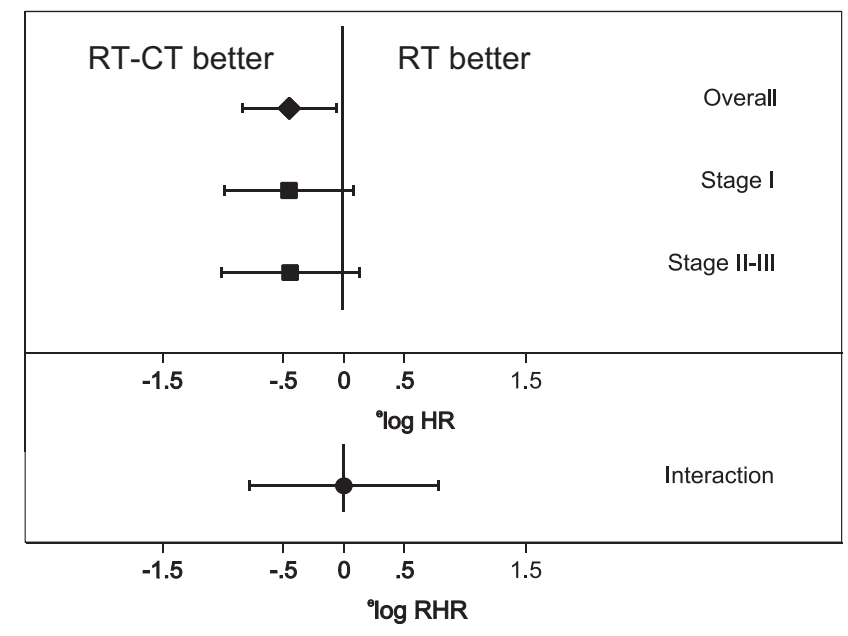

Cell type

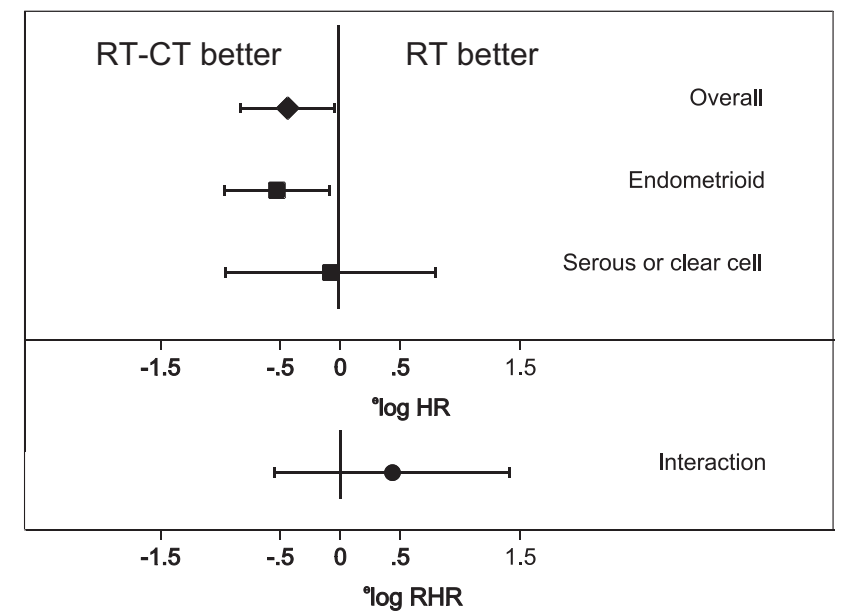

Lymphadenectomy

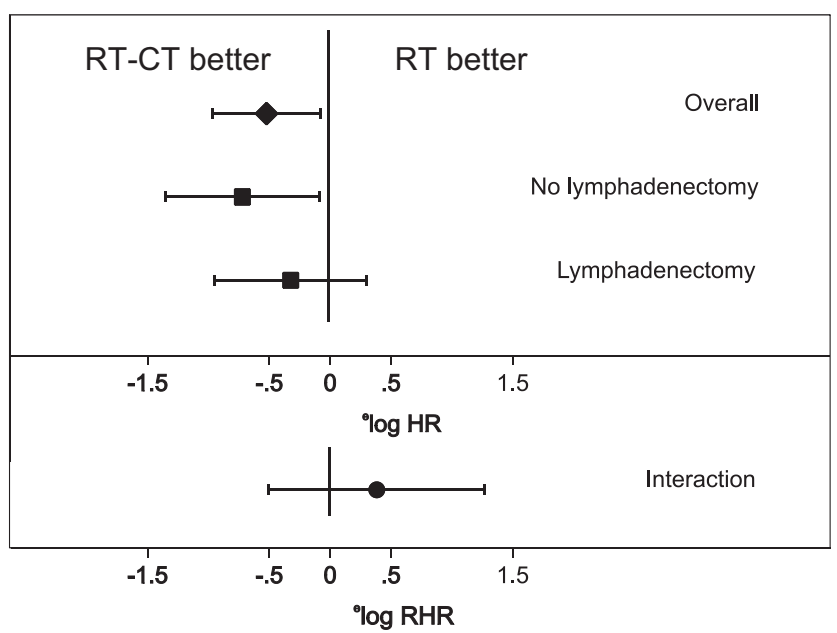

Fig. 4 - Forest plots for interaction between prognostic factors and treatment. The analysis was performed on 444 patients with no missing values for all covariates with progression-free survival (PFS) as the end-point. The analysis of lymphadenectomy was performed on 286 patients with information about lymphadenectomy. The upper bar in each diagram depicts the overall hazard ratio (HR) and the two middle bars show the HR by covariate group. The lowest bar shows the ratio of hazard ratios (RHR), which is a measure of interaction; if it crosses the vertical line there is no significant interaction, which is the case for all five covariates (RT: radiotherapy, RT-CT: sequential radiotherapy and chemotherapy). 


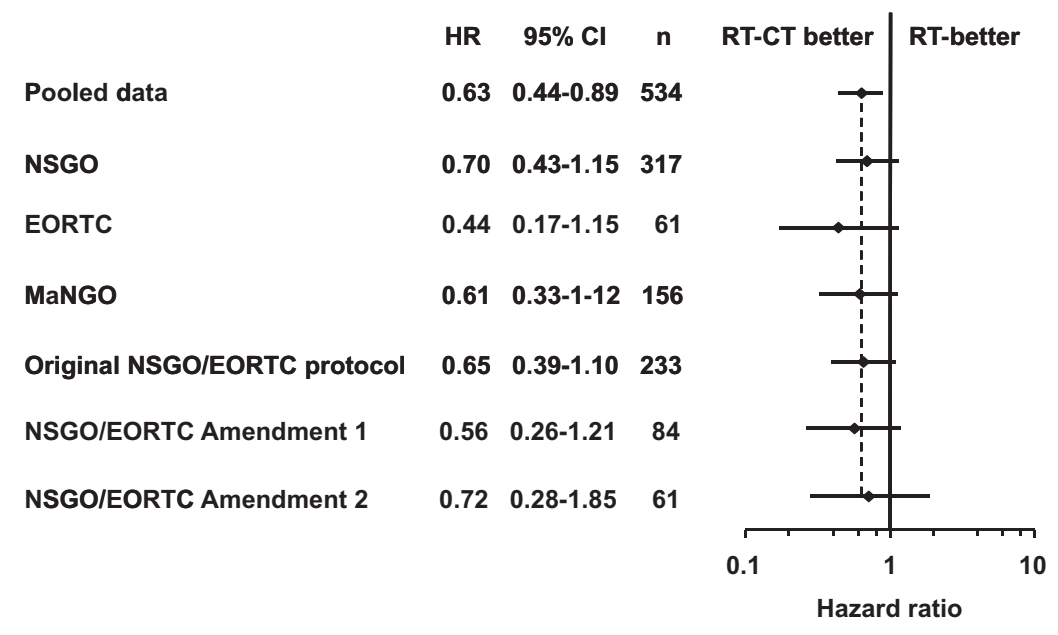

Fig. 5 - Forest plot with progression-free survival (PFS) as end-point illustrating possible heterogeneity depending on study group (NSGO, EORTC or MaNGO) and original protocol, amendment 1 or 2 in the NSGO/EORTC-trial (CI: confidence interval, HR: hazard ratio, RT: radiotherapy and RT-CT: sequential radiotherapy and chemotherapy).

I/stage II-III, patients aged $\leqslant 60 /$ older, grade $1-2 / 3$ or LA performed or not). It is interesting to note that for serous/clear cell carcinomas, the treatment effect was negligible, although with wide confidence intervals. The same tendency could be seen in GOG-122, ${ }^{16}$ where the HR for death in the 83 women with serous carcinomas was slightly above 1.0 in contrast to the HR of 0.48 favouring CT for the endometrioid cell types. Chemotherapy is often recommended for patients with serous/clear cell carcinomas. However, neither the present study nor GOG-122 supports that recommendation. On the other hand, none of the studies can rule out an effect.

We have shown that RT-CT seems superior to RT alone. However, many important questions remain and need to be clarified by future studies. The ongoing International Postoperative Radiotherapy in Endometrial Cancer study (PORTEC-3) compares adjuvant pelvic RT with concomitant cisplatin followed by four courses of paclitaxel + carboplatin (modified from the The Radiation Therapy Oncology Group (RTOG) trial ${ }^{19}$ ) with standard RT. Should the PORTEC-trial confirm the superiority of the combined modality strategy it will still be unsettled as to how much RT adds to CT. Before combining two toxic therapies a study comparing RT-CT versus CT alone should be done. The NSGO has made a proposal (After 4) in the International Gynaecologic Cancer Intergroup (GCIG) setting comparing four courses of paclitaxel + carboplatin followed by RT versus six courses of paclitaxel + carboplatin.

\section{Conflict of interest statement}

None declared.

\section{Acknowledgements}

This study was supported by the Nordic Cancer Union (Grant No. 060004 to NSGO), Fondazione Mattioli to MaNGO and the National Cancer Institute at Bethesda, MD, USA (Grant Nos. 5U10 CA11488-30 through 5U10 CA011488-39 to EORTC). The funding organisations had no influence on the study design; in the collection, analysis and interpretation of data; in the writing of the report; and in the decision to submit the paper for publication.

We thank all the women who participated in this trial and the research staff that helped to recruit patients and to provide data. We also thank the Clinical Trial Unit, Department of Oncology, Linköping University Hospital; the Regional Tumour Registries of Southern and Southeastern Sweden; the EORTC Headquarters for data management in the NSGO/EORTC-study; and the data centre at the Mario Negri Institute for data management in the MaNGO- trial.

Other trial collaborators, listed alphabetically by name, include: Antonio Casada-Herreaz, Department of Medical Oncology, University Hospital, San Carlos, Madrid, Spain; Paul Chinet-Charrot, Department of Oncology, Centre Henri Bequerel, Rouen, France; Stefano Greggi, Istitituto Nazionale per lo Studio e Cure dei Tumori, Napoli, Italy; Jan Jobsen, Medish Spectrum Twente, Enschede, The Netherlands; Angel J. Lacave, Department of Medical Oncology, Hospital General de Asturias, Oviedo, Spain; Christian Marth, Department of Gynecology and Obstetrics, University Hospital Innsbruck, Insbruck, Austria; Saverio Tateo, Fondazione Policlinico S. Matteo di Pavia, Pavia, Italy; and Päivi Vuolo-Merilä, Department of Obstetrics and Gynecology, University Hospital, Oulu, Finland.

Clinical trial registration: The NSGO/EORTC-study is registered in the European Clinical Trials Database with EudraCT No. 2004-002429-37 and in ClinTrials.gov with ID NCT 00005583 and registration date 02/05/2000. The MaNGO-trial is registered in the Italian National Monitoring Centre for Clinical Trials <http://oss-sper-clin.agenziafarmaco.it/project.htm> trial code "ILIADE".

R E F E R E N C E S

1. Parkin DM, Bray F, Ferlay J, Pisani P. Global cancer statistics, 2002. CA Cancer J Clin 2005;55:74-108.

2. Creasman WT, Odicino F, Maisonneuve P, et al. Carcinoma of the corpus uteri. FIGO 26th Annual Report on the Results of 
Treatment in Gynecological Cancer. Int J Gynaecol Obstet 2006;95(Suppl 1):S105-43.

3. Aalders JG, Abeler VM, Kolstad P, Onsrud M. Postoperative external irradiation and prognostic parameters in stage I endometrial carcinoma. Obstet Gynecol 1980;56:419-26.

4. Creutzberg CL, van Putten WLJ, Koper PCM, et al. Surgery and postoperative radiotherapy versus surgery alone for patients with stage-1 endometrial carcinoma: multicentre randomised trial. Lancet 2000;355:1404-11.

5. Keys HM, Roberts JA, Brunetto VL, et al. A phase III trial of surgery with or without adjunctive external pelvic radiation therapy in intermediate risk endometrial adenocarcinoma: a Gynecologic Oncology Group study. Gynecol Oncol 2004;92:744-51.

6. Blake P, Swart AM, Orton J, et al. Adjuvant external beam radiotherapy in the treatment of endometrial cancer (MRC ASTEC and NCIC CTG EN.5 randomised trials): pooled trial results, systematic review, and meta-analysis. Lancet 2009;373:137-46.

7. Hogberg T, Rosenberg P, Kristensen G, et al. A randomized phase-III study on adjuvant treatment with radiation (RT) \pm chemotherapy (CT) in early stage high-risk endometrial cancer (NSGO-EC-9501/EORTC 55991). J Clin Oncol 2007;25(18S) [abstract 5503].

8. Thigpen T, Blessing J, Homesley H, et al. Phase III trial of doxorubicin \pm cisplatin in advanced or recurrent endometrial carcinoma: a Gynecologic Oncology Group (GOG) study. Proc Am Soc Clin Oncol 1993;12 (abstract 830).

9. Pocock SJ, Simon R. Sequential treatment assignment with balancing for prognostic factors in the controlled clinical trial. Biometrics 1975;31:103-15.

10. Signorelli M, Lissoni AA, Cormio G, et al. Modified radical hysterectomy versus extrafascial hysterectomy in the treatment of stage I endometrial cancer: results from the ILIADE randomized study. Ann Surg Oncol 2009;16:3431-41.
11. Benedetti PP, Basile S, Maneschi F, et al. Systematic pelvic lymphadenectomy vs. no lymphadenectomy in early-stage endometrial carcinoma: randomized clinical trial. J Natl Cancer Inst 2008;100:1707-16.

12. Cox DR. Regression models and life tables. J Royal Statist Soc 1972;34:187-220.

13. Barthel FM-S, Royston P. Graphical representation of interactions. Stata J 2006;6:358-63.

14. Scholten AN, van Putten WL, Beerman H, et al. Postoperative radiotherapy for stage 1 endometrial carcinoma: long-term outcome of the randomized PORTEC trial with central pathology review. Int J Radiat Oncol Biol Phys 2005;63:834-8.

15. Morrow CP, Bundy BN, Homesley HD, et al. Doxorubicine as an adjuvant following surgery and radiation therapy in patients with high-risk endometrial carcinoma, stage I and occult stage II: a Gynecologic Oncology Group study. Gynecol Oncol 1990;36:166-71.

16. Randall ME, Filiaci VL, Muss H, et al. Randomized phase III trial of whole-abdominal irradiation versus doxorubicin and cisplatin chemotherapy in advanced endometrial carcinoma: a Gynecologic Oncology Group study. J Clin Oncol 2006;24:36-44.

17. Maggi R, Lissoni A, Spina F, et al. Adjuvant chemotherapy vs radiotherapy in high-risk endometrial carcinoma: results of a randomised trial. Brit J Cancer 2006;95:266-71.

18. Susumu N, Sagae S, Udagawa Y, et al. Randomized phase III trial of pelvic radiotherapy versus cisplatin-based combined chemotherapy in patients with intermediate- and high-risk endometrial cancer: a Japanese Gynecologic Oncology Group study. Gynecol Oncol 2008;108:226-33.

19. Greven K, Winter K, Underhill K, et al. Final analysis of RTOG 9708: adjuvant postoperative irradiation combined with cisplatin/paclitaxel chemotherapy following surgery for patients with high-risk endometrial cancer. Gynecol Oncol 2006;103:155-9. 